\title{
gु
}

\section{Chiral Symmetry Breaking in QCD with Two Light Flavors}

\author{
Georg P. Engel, ${ }^{1}$ Leonardo Giusti, ${ }^{1}$ Stefano Lottini, ${ }^{2}$ and Rainer Sommer ${ }^{2}$ \\ ${ }^{1}$ Dipartimento di Fisica, Università di Milano-Bicocca, and INFN, Sezione di Milano-Bicocca, \\ Piazza della Scienza 3, I-20126 Milano, Italy \\ ${ }^{2}$ John von Neumann Institute for Computing (NIC), DESY, Platanenallee 6, D-15738 Zeuthen, Germany \\ (Received 25 June 2014; revised manuscript received 25 November 2014; published 16 March 2015)
}

A distinctive feature of the presence of spontaneous chiral symmetry breaking in QCD is the condensation of low modes of the Dirac operator near the origin. The rate of condensation must be equal to the slope of $M_{\pi}^{2} F_{\pi}^{2} / 2$ with respect to the quark mass $m$ in the chiral limit, where $M_{\pi}$ and $F_{\pi}$ are the mass and the decay constant of the Nambu-Goldstone bosons. We compute the spectral density of the (Hermitian) Dirac operator, the quark mass, the pseudoscalar meson mass, and decay constant by numerical simulations of lattice QCD with two light degenerate Wilson quarks. We use lattices generated by the Coordinated Lattice Simulation (CLS) group at three values of the lattice spacing in the range 0.05-0.08 fm, and for several quark masses corresponding to pseudoscalar mesons masses down to $190 \mathrm{MeV}$. Thanks to this coverage of parameters space, we can extrapolate all quantities to the chiral and continuum limits with confidence. The results show that the low quark modes do condense in the continuum as expected by the Banks-Casher mechanism, and the rate of condensation agrees with the GellMann-Oakes-Renner relation. For the renormalization-group-invariant ratios we obtain $\left[\Sigma^{\mathrm{RGI}}\right]^{1 / 3} / F=$ $2.77(2)(4)$ and $\Lambda^{\overline{\mathrm{MS}}} / F=3.6(2)$, which correspond to $\left[\Sigma^{\overline{\mathrm{MS}}}(2 \mathrm{GeV})\right]^{1 / 3}=263(3)(4) \mathrm{MeV}$ and $F=$ $85.8(7)(20) \mathrm{MeV}$ if $F_{K}$ is used to set the scale by supplementing the theory with a quenched strange quark.

Introduction.-There is overwhelming evidence that the chiral symmetry group $S U\left(N_{f}\right)_{L} \times S U\left(N_{f}\right)_{R}$ of quantum chromodynamics (QCD) with a small number $N_{f}$ of light flavors breaks spontaneously to $S U\left(N_{f}\right)_{L+R}$. This progress became possible over the last decade thanks to the impressive speed-up of the numerical simulations of lattice QCD with light dynamical fermions; see Ref. [1] for a recent review and a comprehensive list of references. The impact on phenomenological analyses of chiral dynamics is already striking [2].

The formation of a nonzero chiral condensate in the theory, $\Sigma=-\left.\frac{1}{2}\langle\bar{\psi} \psi\rangle\right|_{m=0}$, was conjectured to be the effect of the condensation of the low modes of the Dirac operator near the origin [3]. The rate of condensation is indeed a renormalizable universal quantity in QCD, and is unambiguously defined once the bare parameters in the action of the theory have been renormalized [4]. The Banks-Casher mechanism links the spectral density $\rho(\lambda, m)$ of eigenvalues $i \lambda$ of the Dirac operator to the condensate as [3]

$$
\lim _{\lambda \rightarrow 0} \lim _{m \rightarrow 0} \lim _{V \rightarrow \infty} \rho(\lambda, m)=\frac{\Sigma}{\pi}
$$

an identity which can be read in both directions; a nonzero spectral density implies that the symmetry is broken by a nonvanishing $\Sigma$ and vice versa.

The above conceptual and technical advances in lattice gauge theory paved the way for a quantitative study of the Banks-Casher mechanism from first principles. It is the aim of this Letter to achieve a precise and reliable determination of the density of eigenvalues of the Euclidean Dirac operator $D$ near the origin at small quark masses in the continuum. As in any numerical computation, the limits in Eq. (1) inevitably require an extrapolation of the results with a predefined functional form. The distinctive signature for spontaneous symmetry breaking is the agreement between the chiral-limit value of the spectral density at the origin, reached by extrapolating the data with the functional form dictated by chiral perturbation theory (ChPT), and the slope of $M_{\pi}^{2} F_{\pi}^{2} / 2$ with respect to the quark mass $m[5,6]$. We thus complement our study with the computations of $m, M_{\pi}$, and $F_{\pi}$.

To reach these goals, we use $O(a)$-improved Wilson fermions at several lattice spacings, and we extrapolate the numerical results to the universal continuum limit following the Symanzik effective theory analysis. For technical reasons we focus on the mode number of the Dirac operator [4]

$$
\nu(\Lambda, m)=V \int_{-\Lambda}^{\Lambda} d \lambda \rho(\lambda, m)
$$

which at the same time is the average number of eigenmodes of the massive Hermitian operator $D^{\dagger} D+m^{2}$ with eigenvalues $\alpha \leq M^{2}=\Lambda^{2}+m^{2}$. It is a renormalizationgroup-invariant (RGI) quantity as it stands. Its (normalized) discrete derivative 


$$
\tilde{\rho}\left(\Lambda_{1}, \Lambda_{2}, m\right)=\frac{\pi}{2 V} \frac{\nu\left(\Lambda_{2}\right)-\nu\left(\Lambda_{1}\right)}{\Lambda_{2}-\Lambda_{1}}
$$

carries the same information as $\rho(\lambda, m)$, but the effective density $\tilde{\rho}$ is a more convenient quantity to consider in numerical computations. For practical purposes we also extend the theory by introducing a quenched "strange" quark so to have a graded chiral symmetry group $S U(3 \mid 1)$. This is instrumental to derive the ChPT formula for $\rho(\lambda, m)$ [7], and allows us to fix the lattice spacing from the kaon decay constant $F_{K}$. The latter is a well-defined intermediate reference scale which can be computed precisely on the lattice [8] and is directly accessible to experiments once the Cabibbo-Kobayashi-Maskawa (CKM) matrix element $\left|V_{u s}\right|$ is known. This scale is used here to convert all quantities in physical units, with the scheme-dependent ones $(m, \rho, \Lambda, \Sigma)$ renormalized in the $\overline{\mathrm{MS}}$ scheme at $\mu=2 \mathrm{GeV}$. The final results, however, are independent of this intermediate step: they are expressed as ratios of quantities of the two-flavor theory only.

It is worth noting that there were several exploratory studies of the spectral density of the Dirac operator in QCD; see for instance $[4,9,10]$. The approach pursued here is rather general (preliminary results were presented in Ref. [11]), and it may be useful in order to study theories at nonzero temperature or strongly interacting models of electroweak symmetry breaking [12].

Lattice computation.-We have profited from CLS simulations of two-flavor QCD with the $O(a)$-improved Wilson action. On all the lattices listed in Table I we have computed the mode number and the two-point functions of $\bar{\psi}_{1} \gamma_{5} \psi_{2}$ and $\bar{\psi}_{1} \gamma_{0} \gamma_{5} \psi_{2}$. The ensembles have lattice spacings of $a=0.075,0.065$, and $0.048 \mathrm{fm}$ as measured from $F_{K}$ [8]. The quark masses range from 6 to $40 \mathrm{MeV}$ and are small compared to the typical scale of the theory from the condensate or the string tension of about $250-450 \mathrm{MeV}$. All lattices are of size $2 L \times L^{3}$, and the pion mass is always

TABLE I. Overview of the ensembles used in this study. We give label, spatial extent of the lattice, quark mass $m$, pion mass $M_{\pi}$, and its decay constant $F_{\pi}$, and the (updated) value of the lattice spacing determined from $F_{K}$ as in Ref. [8].

\begin{tabular}{lcccccc}
\hline \hline id & $L / a$ & $m[\mathrm{MeV}]$ & $F_{\pi}[\mathrm{MeV}]$ & $M_{\pi}[\mathrm{MeV}]$ & $M_{\pi} L$ & $a[\mathrm{fm}]$ \\
\hline A3 & 32 & $37.4(9)$ & $120.8(7)$ & $496(6)$ & 6.0 & $0.0749(8)$ \\
A4 & 32 & $22.8(6)$ & $110.7(6)$ & $386(5)$ & 4.7 & \\
A5 & 32 & $16.8(4)$ & $106.0(6)$ & $333(5)$ & 4.0 & \\
B6 & 48 & $12.2(3)$ & $102.3(5)$ & $283(4)$ & 5.2 & \\
E5 & 32 & $32.0(8)$ & $115.2(6)$ & $440(5)$ & 4.7 & $0.0652(6)$ \\
F6 & 48 & $16.5(4)$ & $105.3(6)$ & $314(3)$ & 5.0 & \\
F7 & 48 & $12.0(3)$ & $100.9(4)$ & $268(3)$ & 4.3 & \\
G8 & 64 & $6.1(2)$ & $95.8(4)$ & $193(2)$ & 4.1 & \\
N5 & 48 & $34.8(8)$ & $115.1(7)$ & $443(4)$ & 5.2 & $0.0483(4)$ \\
N6 & 48 & $20.9(5)$ & $105.8(5)$ & $342(3)$ & 4.0 & \\
O7 & 64 & $12.9(3)$ & $101.2(4)$ & $269(3)$ & 4.2 & \\
\hline \hline
\end{tabular}

large enough so that $m_{\pi} L \geq 4$. Finite-size effects are within the statistical errors for all measured quantities; see Ref. [13] for more details. The error analysis takes care of autocorrelations [14], all Markov chains except for one (N5) being of length between 24 and $74 \tau_{\text {exp }}$, with $\tau_{\exp }$ being the longest autocorrelation time measured on the chain.

The mode number has been computed for nine values of $\Lambda$ in the range $20-120 \mathrm{MeV}$ with a statistical accuracy of a few percent on all lattices. Four larger values of $\Lambda$ in the range 150-500 MeV have also been analyzed for the ensemble E5; see Ref. [13] for tables with all results. In Fig. 1 (top-left) we show $\nu$ as a function of $\Lambda$ for the lattice O7, corresponding to the smallest quark mass at the smallest lattice spacing. On all other lattices an analogous qualitative behavior is observed. The mode number is a nearly linear function in $\Lambda$ up to approximately 100$150 \mathrm{MeV}$. A clear departure from linearity is observed for $\Lambda>200 \mathrm{MeV}$ on the lattice E5. At the percent precision, however, the data show statistically significant deviations from the linear behavior already below $100 \mathrm{MeV}$. To guide the eye, a quadratic fit in $\Lambda$ is shown in Fig. 1, and the values of the coefficients are given in the caption. The bulk of $\nu$ is given by the linear term, while the constant and the quadratic term represent $O(10 \%)$ corrections in the fitted range. The nearly linear behavior of the mode number, expected if the Banks-Casher mechanism is at work, is manifest on the top-right plot of Fig. 1, where the cubic root of the discrete derivative defined in Eq. (3) is shown as a function of $\Lambda=\left(\Lambda_{1}+\Lambda_{2}\right) / 2$ for each couple of consecutive values of $\Lambda$. When the regularization breaks chiral symmetry, discretization effects heavily distort the spectral density near $\lambda=0[15,16]$; we thus focus on the effective spectral density rather than the mode number.

In general, $\tilde{\rho}^{1 / 3}$ shows quite a flat behavior in $\Lambda$ at fine lattice spacings and light quark masses, similar to the one shown in Fig. 1 (top-right). Because the action and the mode number are $O(a)$ improved, the Symanzik effectivetheory analysis predicts that discretization errors start at $O\left(a^{2}\right)$ [4]. In order to remove them, we interpolate the effective spectral density to three quark mass values ( $m=12.9,20.9$, and $32.0 \mathrm{MeV}$ ) at each lattice spacing. The values of $\tilde{\rho}^{1 / 3}$ show very mild discretization effects at light $m$ and $\Lambda$, while they differ by up to $15 \%$ among the three lattice spacings toward heavier $\Lambda$. Within the statistical errors, all data sets are compatible with a linear dependence in $a^{2}$, and we thus independently extrapolate each triplet of points to the continuum limit accordingly. The difference between the values of $\tilde{\rho}^{1 / 3}$ at the finest lattice spacing and the continuum-extrapolated ones is within $5 \%$ for the lightest $m$ and $\Lambda$, and it remains within few standard deviations at heavier values of $m$ and $\Lambda$. This makes us confident that the extrapolation removes the cutoff effects within the errors quoted. 

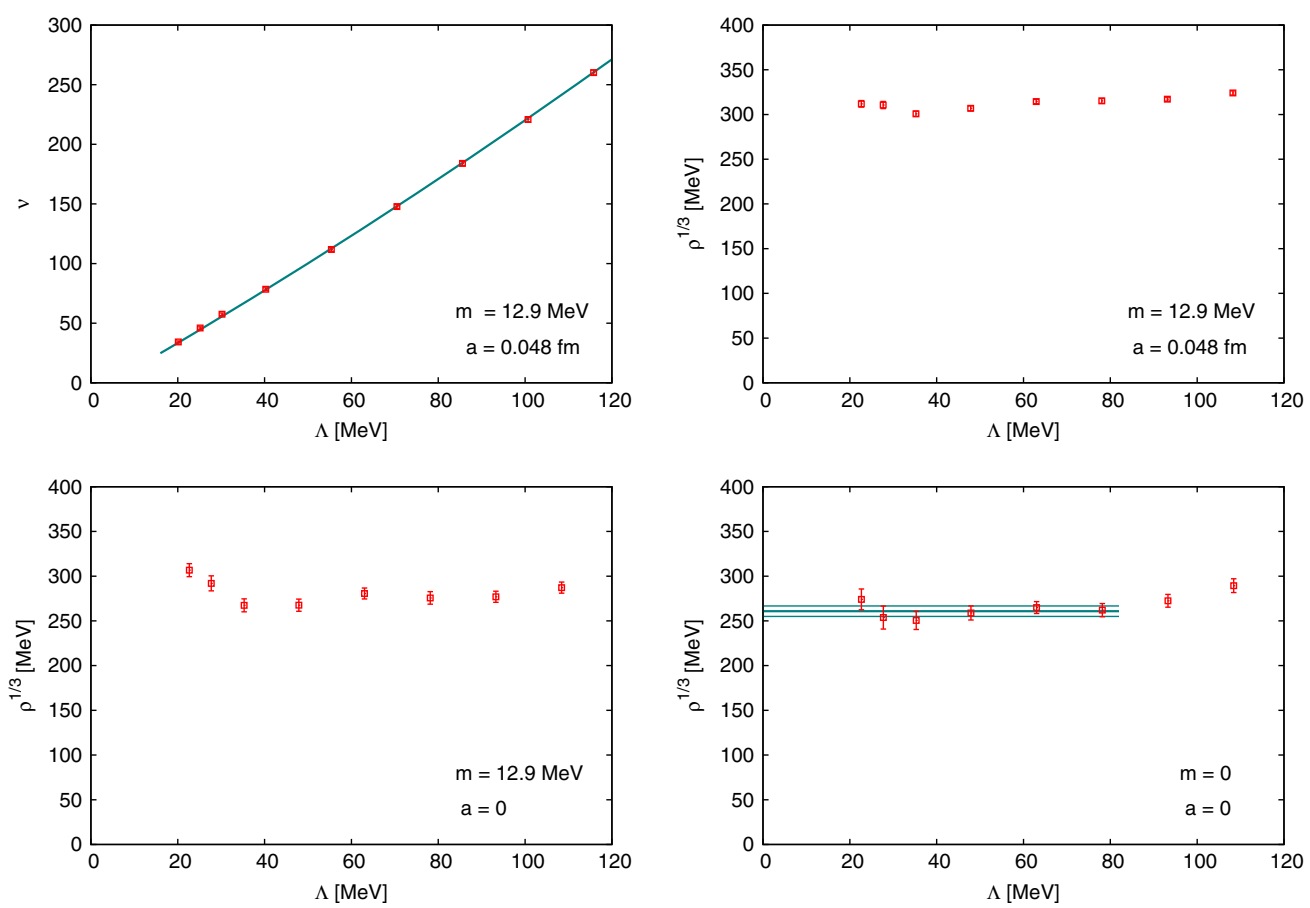

FIG. 1 (color online). Top-left: The mode number as a function of $\Lambda$ for O7; a quadratic fit of the data ( $\Lambda$ in MeV) gives $\nu=-9.0(13)+2.07(7) \Lambda+0.0022(4) \Lambda^{2}$. Top-right: $\tilde{\rho}^{1 / 3}$ for the same ensemble as a function of $\Lambda=\left(\Lambda_{1}+\Lambda_{2}\right) / 2$. Bottom-left: $\tilde{\rho}^{1 / 3}$ in the continuum limit at the smallest reference quark mass. Bottom-right: $\tilde{\rho}^{1 / 3}$ in the continuum and chiral limit. Note the flat dependence on $\Lambda$ which agrees with the expectation from NLO ChPT; a plateau fit in the interval 20-80 MeV is also shown.

The results for $\tilde{\rho}^{1 / 3}$ at $m=12.9 \mathrm{MeV}$ in the continuum limit are shown as a function of $\Lambda$ in the bottom-left plot of Fig. 1. A similar $\Lambda$ dependence is observed at the two other reference masses. It is noteworthy that no assumption on the presence of spontaneous symmetry breaking was needed so far. These results point to the fact that the spectral density of the Dirac operator in two-flavor QCD is nonzero and (almost) constant in $\Lambda$ near the origin at small quark masses. This is consistent with the expectations from the Banks-Casher mechanism. In the presence of spontaneous symmetry breaking, next-to-leading (NLO) ChPT indeed predicts $[4,7,17,18]$

$$
\begin{aligned}
\tilde{\rho}^{\text {nlo }}= & \Sigma\left\{1+\frac{m \Sigma}{(4 \pi)^{2} F^{4}}\left[3 \bar{l}_{6}+1-\ln (2)\right.\right. \\
& \left.\left.-3 \ln \left(\frac{\Sigma m}{F^{2} \bar{\mu}^{2}}\right)+\tilde{g}_{\nu}\left(\frac{\Lambda_{1}}{m}, \frac{\Lambda_{2}}{m}\right)\right]\right\},
\end{aligned}
$$

i.e., an almost flat function in (small) $\Lambda$ at (small) finite quark masses. The parameter $\bar{l}_{6}$ is a low-energy constant of the $S U(3 \mid 1)$ chiral effective theory renormalized at the scale $\bar{\mu}$, while $\tilde{g}_{\nu}$ is a parameter-free function; see Ref. [13]. Once the pion mass and decay constant are measured, the (mild) parameter-free $\Lambda$ dependence of $\tilde{\rho}^{\text {nlo }}$ in Eq. (4) is compatible with our data.

The extrapolation to the chiral limit requires an assumption on how the effective spectral density $\tilde{\rho}$ behaves when $m \rightarrow 0$. In this respect it is interesting to notice that toward the chiral limit, the function in Eq. (4) is the simplest possible one; i.e., it goes linearly in $m$ since there are no chiral logarithms at fixed $\Lambda$ [4]. A fit of the data to Eq. (4) shows that they are compatible with that NLO formula. Equation (4) predicts that in the chiral limit $\tilde{\rho}^{\text {nlo }}=\Sigma$ also at nonzero $\Lambda$, since all NLO corrections vanish [18]. By extrapolating the effective spectral density with Eq. (4) but allowing for the constant term to depend on $\Lambda$, we obtain the results shown in the bottom-right plot of Fig. 1 with a $\chi^{2} /$ d.o.f. $=16.4 / 14$. Within errors, the $\Lambda$ dependence is clearly compatible with a constant up to $\approx 100 \mathrm{MeV}$. Moreover, the differences between the values of $\tilde{\rho}^{1 / 3}$ in the chiral limit and those at $m=12.9 \mathrm{MeV}$ are of the order of the statistical error; i.e., the extrapolation is very mild. A fit to a constant of the data gives $\Sigma^{1 / 3}=261(6)(8) \mathrm{MeV}$, where the first error is statistical and the second one is systematic. The latter is a conservative estimate obtained by performing various combined fits of all data suggested by NLO ChPT and the Symanzik effective theory analysis [13].

To compare the value of the spectral density at the origin with the slope of $M_{\pi}^{2} F_{\pi}^{2} / 2$ with respect to the quark mass $m$, we complement the computation of the mode number with those for the pion masses and the decay constants, $M_{\pi}$ and $F_{\pi}$, as well as the quark mass $m$. They are extracted from the two-point functions of the nonsinglet pseudoscalar density and axial current as in Refs. [8,19]; see Ref. [13] for more details. Note that the quark mass, 


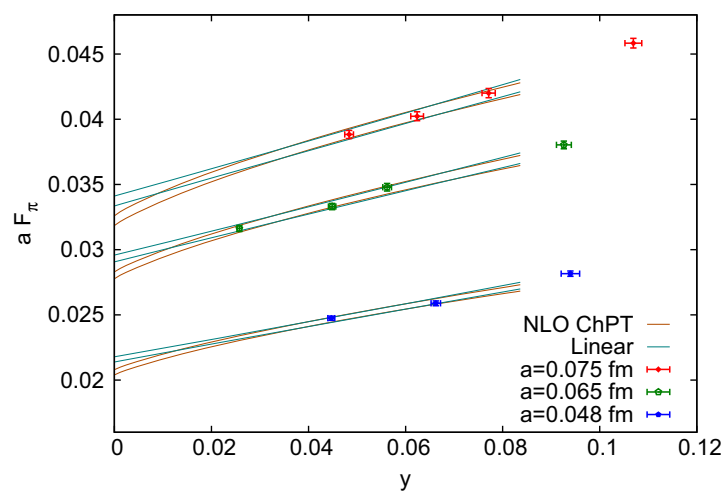

FIG. 2 (color online). The pseudoscalar decay constant $a F_{\pi}$ versus $y=M_{\pi}^{2} /\left(4 \pi F_{\pi}\right)^{2}$. The bands are the result of a combined fit; see main text.

denoted by $m$ here, is indicated by $m_{\mathrm{R}}$ in Ref. [13]. The results are reported in Table I, and those for the pseudoscalar decay constant in lattice units are shown in Fig. 2 versus $y=M_{\pi}^{2} /\left(4 \pi F_{\pi}\right)^{2}$. We fit $F_{\pi}$ to the function

$$
a F_{\pi}=a F(a)\{1-y \ln (y)+b y\},
$$

where $b$ is common to all lattice spacings, restricted to the points with $M_{\pi}<400 \mathrm{MeV}$ (see Fig. 2). Apart from the NLO ChPT just given, we also perform a number of alternative extrapolations in $y$. As a final result we quote $a F(a)=0.0330(4)(8), 0.0287(3)(7)$, and 0.0211(2)(5) at $a=0.075,0.065$, and $0.048 \mathrm{fm}$, respectively, where the second (systematic) error takes into account the spread of the results from the various fits. By performing a continuum-limit extrapolation we obtain our final result $F=85.8(7)(20) \mathrm{MeV}$. Once the value of $F$ is determined, we compute the ratio $M_{\pi}^{2} / 2 m$ for all data points. We fit the data with $M_{\pi}<400 \mathrm{MeV}$ to

$$
\left[\frac{M_{\pi}^{2}}{2 m F}\right]^{1 / 3}=\left[s_{0}+s_{1}(a F)^{2}\right]\left\{1+\frac{y}{6} \ln (y)+d y\right\},
$$

where $s_{0}, s_{1}$, and $d$ are free parameters common to all lattice spacings. Also, in this case we checked several variants although the data look very flat up to the heaviest mass. From the fit we get $s_{0}=3.06(3)(4)$ which, together with the value of $F$, leads to $\left[\sum_{\mathrm{GMOR}}^{\mathrm{MS}}(2 \mathrm{GeV})\right]^{1 / 3}=$ 263(3)(4) MeV, where errors are determined as for $F$.

Discussion and conclusions.-From the previous analysis, our best results for the leading-order low-energy constants of QCD with two flavors are

$$
\begin{aligned}
{\left[\Sigma^{\overline{\mathrm{MS}}}(2 \mathrm{GeV})\right]^{1 / 3} } & =263(3)(4) \mathrm{MeV}, \\
F & =85.8(7)(20) \mathrm{MeV} .
\end{aligned}
$$

By updating the value of the $\Lambda$ parameter in Refs. $[8,20]$ to $\Lambda^{\mathrm{MS}}=311(19) \mathrm{MeV}$ and by taking into account the correlation with $F$, we obtain the dimensionless ratios

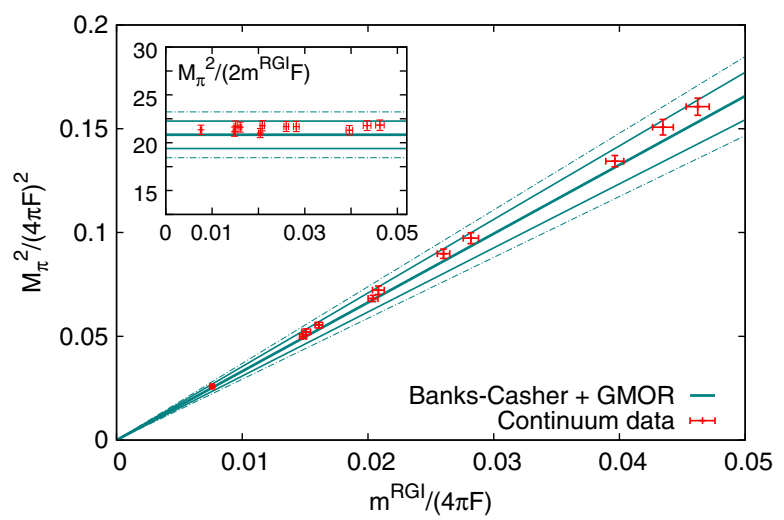

FIG. 3 (color online). The pion mass squared versus the RGI quark mass $\left(m / m^{\mathrm{RGI}}=0.740(12)[8]\right)$, both normalized to $4 \pi F$ which is roughly $1 \mathrm{GeV}$. The ratio $\left(M_{\pi}^{2} / 2 m^{\mathrm{RGI}} F\right)^{1 / 3}$ is the continuum value obtained by extrapolating the data with Eq. (6). The central line is the GMOR contribution to the pion mass squared, computed by taking the direct measure of the condensate from the spectral density. The upper and lower solid lines show the statistical error and the dotted-dashed ones the total error, the systematic being added in quadrature.

$$
\frac{\left[\Sigma^{\mathrm{RGI}}\right]^{1 / 3}}{F}=2.77(2)(4), \quad \frac{\Lambda^{\mathrm{MS}}}{F}=3.6(2) .
$$

where the RGI condensate is defined with the convention of Refs. [21,22].

Our results show that the spectral density of the Dirac operator in the continuum is nonzero at the origin and that its value agrees with the slope of $M_{\pi}^{2} F_{\pi}^{2} / 2$ with respect to the quark mass when both are extrapolated to the chiral limit. If expanded in $m, M_{\pi}^{2}$ is dominated by the leading Gell-Mann-Oakes-Renner term proportional to the chiral condensate; see Fig. 3. The ratio $M_{\pi}^{2} / 2 m$ is nearly constant within errors up to quark masses that are about 1 order of magnitude larger than in nature.

Measurements have been performed on BlueGene/Q at CINECA (CINECA-INFN agreement, ISCRA project IsB08_Condnf2), on HLRN, on JUROPA/JUQUEENat Jülich JSC, on PAX at DESY, Zeuthen, and on Wilson at Milano-Bicocca. We thank these institutions for the computer resources and the technical support. We are grateful to our colleagues within the CLS initiative for sharing the ensembles of gauge configurations. G. P. E. and L. G. acknowledge partial support by the MIUR-PRIN Contract No. 20093BMNNPR. S. L. and R. S. acknowledge support by the DFG Sonderforschungsbereich/ Transregio SFB/TR9.

[1] S. Schaefer, Proc. Sci, LATTICE2012 (2012) 001.

[2] H. Leutwyler, International School of Subnuclear Physics: 45th Course: Searching for the "Totally Unexpected" in the LHC Era, Erice, 2007 (unpublished). 
[3] T. Banks and A. Casher, Nucl. Phys. B169, 103 (1980).

[4] L. Giusti and M. Luscher, J. High Energy Phys. 03 (2009) 013.

[5] S. Weinberg, Physica (Amsterdam) 96A, 327 (1979).

[6] J. Gasser and H. Leutwyler, Nucl. Phys. B250, 465 (1985).

[7] J. Osborn, D. Toublan, and J. Verbaarschot, Nucl. Phys. B540, 317 (1999).

[8] P. Fritzsch, F. Knechtli, B. Leder, M. Marinkovic, S. Schaefer, R. Sommer, and F. Virotta, Nucl. Phys. B865, 397 (2012).

[9] H. Fukaya, S. Aoki, T. W. Chiu, S. Hashimoto, T. Kaneko, J. Noaki, T. Onogi, and N. Yamada (JLQCD Collaboration and TWQCD Collaboration), Phys. Rev. D 83, 074501 (2011).

[10] K. Cichy, E. Garcia-Ramos, and K. Jansen, J. High Energy Phys. 10 (2013) 175.

[11] G. P. Engel, L. Giusti, S. Lottini, and R. Sommer, Proc. Sci., LATTICE2013 (2014) 119.

[12] A. Patella, Phys. Rev. D 86, 025006 (2012).
[13] G. P. Engel, L. Giusti, S. Lottini, and R. Sommer, Phys. Rev. D 91, 054505 (2015).

[14] S. Schaefer, R. Sommer, and F. Virotta (ALPHA Collaboration), Nucl. Phys. B845, 93 (2011).

[15] L. Del Debbio, L. Giusti, M. Lüscher, R. Petronzio, and N. Tantalo, J. High Energy Phys. 02 (2006) 011.

[16] P. H. Damgaard, K. Splittorff, and J. J. M. Verbaarschot, Phys. Rev. Lett. 105, 162002 (2010).

[17] H. Leutwyler and A. V. Smilga, Phys. Rev. D 46, 5607 (1992).

[18] A. V. Smilga and J. Stern, Phys. Lett. B 318, 531 (1993).

[19] L. Del Debbio, L. Giusti, M. Luscher, R. Petronzio, and N. Tantalo, J. High Energy Phys. 02 (2007) 082.

[20] M. Della Morte, R. Frezzotti, J. Heitger, J. Rolf, R. Sommer, and U. Wolff, Nucl. Phys. B713, 378 (2005).

[21] S. Capitani, M. Luscher, R. Sommer, and H. Wittig (ALPHA Collaboration), Nucl. Phys. B544, 669 (1999).

[22] M. Della Morte, R. Hoffmann, F. Knechtli, J. Rolf, R. Sommer, I. Wetzorke, and U. Wolff (ALPHA Collaboration), Nucl. Phys. B729, 117 (2005). 